Journal of Animal and Veterinary Advances 11 (14): 2564-2569, 2012

ISSN: $1680-5593$

(C) Medwell Journals, 2012

\title{
The Effects of Rosiglitazone on Fatty Liver of High Fat and Cholesterol-Fed Rabbits
}

\author{
${ }^{1,2,4}$ Guangwei Zhang, ${ }^{1,2}$ Kena Wei, ${ }^{1,2}$ Enqi Liu, ${ }^{1}$ Yanli Wang, ${ }^{1}$ Yafeng Li, \\ ${ }^{1} \mathrm{Qi} \mathrm{Yu},{ }^{1}$ Yulong Chen, ${ }^{1}$ Bingqiao Huang, ${ }^{3}$ Shemin Lu, ${ }^{1}$ Xicai Yan and ${ }^{1,2}$ Sihai Zhao \\ ${ }^{1}$ Laboratory for Lipid Metabolism and Atherosclerosis, Cardiovascular Research Center, \\ $\mathrm{Xi}$ ' an Jiaotong University, Shaanxi, China \\ ${ }^{2}$ Laboratory Animal Center, ${ }^{3}$ Department of Molecular Biology, \\ School of Medicine, Xi' an Jiaotong University, Shaanxi, China \\ ${ }^{4} \mathrm{Xi}$ ' an Medical University, Shaanxi, China
}

\begin{abstract}
What the effects of rosiglitazone treatment on the fatty liver remained controversial. The aim of this study was to investigate the effects of rosiglitazone on fatty liver of rabbits. Thirty nine male Japanese white rabbits were divided into three groups, the rosiglitazone treatment Group $1(3 \mathrm{mg} / \mathrm{kg} / \mathrm{day})$, rosiglitazone treatment Group $2(6 \mathrm{mg} / \mathrm{kg} /$ day $)$ and the control group and were fed a High Fat and Cholesterol Diet (HFCD) for 8 weeks. The Rabbit Fatty Liver Model was successfully established. HFCD resulted in a notable increase in plasma Total Cholesterol (TC) and plasma Triglycerides (TG). However, there was no significant difference among three groups in the plasma and liver parameters of TG and TC. The total body fat weight and the ratio of fat weight to body weight were higher in the rosiglitazone treatment groups vs. the control group while the ratio of the main organs and body weight were lower in the rosiglitazone treatment groups as compared to the control group. Hematoxylin and Eosin $(\mathrm{HE})$ staining of liver suggested that rosiglitazone treatment led to a slight improvement of fatty liver in HFCD rabbits but the difference was not significant. The findings demonstrate that rosiglitazone treatment did not lead to significant improvement of fatty liver in rabbits.
\end{abstract}

Key words: Rabbits, nonalcoholic fatty liver disease, rosiglitazone, peroxisome proliferator-activated receptor, organs, China

\section{INTRODUCTION}

Clinical data indicate that primary Nonalcoholic Fatty Liver Disease (NAFLD) represents not only the most common form of all liver disorders but also the most frequent cause of chronic liver disease in obese patients (Chan et al., 2004). Primary NAFLD is caused by conditions associated with metabolic syndrome such as type 2 diabetes, obesity and hyperlipidemia (Chan et al., 2004; Amarapurka et al., 2006; Chitturi et al., 2002; Marchesini et al., 2003). Non-Alcoholic Steatohepatitis (NASH) is the most extreme form of NAFLD and is regarded as a major cause of cirrhosis of the liver of unknown cause (Clark and Diehl, 2003). There is also no standard therapy recommended for NAFLD. Obesity and insulin resistance are inextricably linked to NAFLD and therefore therapies directed at weight reduction and improved insulin sensitivity have been investigated. Peroxisome Proliferator-Activated Receptors (PPARs) have been found to regulate diverse aspects of lipid metabolism including fatty acid oxidation, fat cell development, lipoprotein metabolism and glucose homeostasis. The PPAR $\gamma$ agonist, rosiglitazone was also reported to attenuate liver inflammation in a rat model of nonalcoholic steatohepatitis (Tahan et al., 2007; Wang et al., 2008). In contrast, rosiglitazone reduced the activity of Complex I and increased oxidative stress and liver steatosis in ob/ob mice (Garcia-Ruiz et al., 2007). Although, rodent models have achieved more progress in the study of effects of liver disease, rabbits may play special role in the study of fatty liver because their lipoprotein metabolism is similar to humans (Fan and Watanabe, 2003; Kawai et al., 2006). In the previous study, researchers also found rosiglitazone treatment led to a lower incidence rate of fatty liver in $0.3 \%$ cholesterol diet-fed rabbits (Zhao et al., 2008a). The effects of rosiglitazone treatment on the high fat and cholesterol diet induced-fatty liver in the rabbit model remain unknown. In this study, researchers attempted to investigate the hypothesis of whether activation of PPAR $y$ by the administration of rosiglitazone could affect the development of fatty liver in rabbits.

Corresponding Author: Sihai Zhao, Laboratory Animal Center, School of Medicine, Xi'an Jiaotong University, 710061 Shaanxi, China 


\section{MATERIALS AND METHODS}

Animals: Japanese white rabbits were provided by the Laboratory Animal Center of Xi'an Jiaotong University. Thirty nine male rabbits were randomly divided into three groups and fed a High Fat and Cholesterol Diet (HFCD containing $1.25 \%$ cholesterol and $10 \%$ corn oil) (control group, $\mathrm{n}=10$ ) or HFCD plus orally administration of

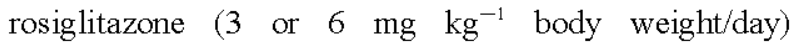
(rosiglitazone treatment group, $\mathrm{n}=13$ for each group) for 8 weeks. The cholesterol dose was chosen based on the previous studies (Otogawa et al., 2007). At the end of the experiment, all rabbits were sacrificed by intravenous injection of an overdose of sodium pentobarbital solution. The experimental protocols were approved by the Xi' an Jiaotong University Laboratory Animal Administration Committee and performed according to the Xi' an Jiaotong University Guidelines for Animal Experimentation.

Plasma lipids and glucose tolerance test: After overnight fasting, blood samples were collected via the auricular artery in tubes containing EDTA every week. Blood samples were stored on ice and centrifuged $(3000 \mathrm{rpm}$, $5 \mathrm{~min}, 4^{\circ} \mathrm{C}$ ) to obtain plasma. Plasma Triglycerides (TG), Total Cholesterol (TC) and glucose levels were measured using commercial assay kits (Beijing Biosino Biotechnology Co., Ltd. China). For the evaluation of glucose metabolism, rabbits were fasted overnight and an Intravenous Glucose Tolerance Test (IVGTT) was performed as beforey described (Liu et al., 2005; Zhao et al., 2008b). A bolus of glucose $\left(0.6 \mathrm{~g} \mathrm{~kg}^{-1}\right.$ body weight) was injected to the ear vein and a blood sample was collected through the ear artery at $5,10,15,20,30,45$, 60,75 and $120 \mathrm{~min}$. Plasma glucose was assayed as described. The incremental Area Under the Curve (AUC) was calculated according to the trapezium rule (Liu et al., 2005).

Isolation and measurement of liver lipids: About $1 \mathrm{~g}$ of liver tissue was homogenized with $19 \mathrm{~mL}$ chloroformmethanol $(2: 1)$ mixture $(\mathrm{v} / \mathrm{v})$ to a final dilution volume of the liver sample, i.e., the liver homogenate from $g$ of tissue was diluted to a volume of $20 \mathrm{~mL}$. The homogenate was extracted overnight $\left(4^{\circ} \mathrm{C}\right)$ and centrifuged $(3000 \mathrm{rpm}$, $20 \mathrm{~min}$ ). Pipetted $100 \mathrm{uL}$ liquids from the lower phase contained essentially all the tissue lipids and were transferred into a glass tube. These liquids were dried with nitrogen to make chloroform diffusion and all of the lipids almost remained in the bottom of the tube (Folch et al., 1957). The lipids were dissolved with $50 \mathrm{uL}$ ethanol and these were used to measure liver TC andTG using commercial assay kits.
Adipose tissue contents: Adipose tissues from the whole body were carefully removed, weighed and the data were expressed as total body fat weight ( $\mathrm{g}$ ) or a percentage of body weight. Researchers divided the adipose tissues into:

- Subcutaneous adipose tissue including fat from the inguinal, axilla and scapular regions

- Visceral adipose tissue from the abdominal cavity, mesenterium and retroperitoneal fat (Marchesini et al., 2003). Researchers compared the adipose weight of each part among the three groups

Histology analysis: After rabbits were sacrificed, the adipose tissue, liver, kidney, spleen, heart and lung were removed, weighed and divided into small pieces then fixed in a $10 \%$ neutral buffered formalin for subsequently histological analysis. These tissues were embedded into paraffin, cut in $4 \mu \mathrm{m}$ sections and stained with Haematoxylin and Eosin ( $\mathrm{HE})$ for histological examination. Researchers compared the proportions of main organs weight to body weight and observed the sections of $\mathrm{HE}$ staining. Frozen sections of liver ( $5 \mu \mathrm{m}$ thick) were stained with $0.5 \%$ Oil Red $O$ for visualization of hepatic fat and then counterstained with hematoxylin (Bai et al., 2011). The severity of fatty liver development was evaluated as diehl described (Diehl et al., 1988). Four grades of liver fatty degeneration were identified based on the percentage of fatty vacuolated liver cell in the total liver cells after histological examination without fatty vacuolated liver cell (-); fatty vacuolated liver cells $<1 / 3$ $(+)$; fatty vacuolated liver cells ranged from $1 / 3-2 / 3$; fatty vacuolated liver cells $>2 / 3(+++)$; all liver cells are fatty vacuolated $(++++)$.

Statistical analysis: Results are expressed as the means \pm SEM. Statistical analysis was performed using either the Student's t-test for data with an equal F-value or Welch's t-test when the F-value was not equal. Rank test was also used for measurement data. A $p<0.05$ was considered statistically significant.

\section{RESULTS AND DISCUSSION}

Plasma parameters, liver parameters and IVGTT: As shown in Fig. 1, the plasma levels of TC and TG were not statistically different between two rosiglitazone-treated groups and control group after feeding HFCD for 8 weeks. Plasma TC and TG levels were measured weekly during the whole period of the experiment however, there was no significant difference among three groups at all time points (Fig. 1a and b). There was also no significant difference in the liver parameters of TG and TC among 

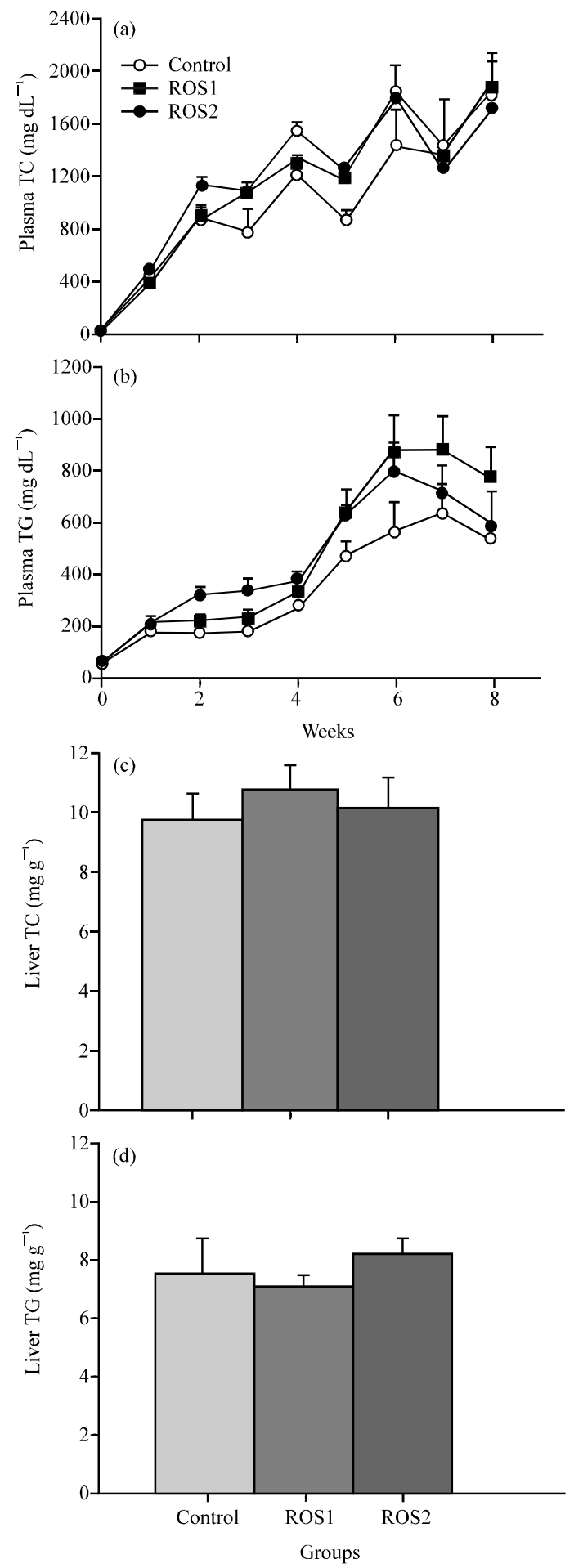

Fig. 1: (a) Plasma TC, (b) plasma TG, (c) liver TC and (d) liver TG. Data are expressed as the mean $\pm \mathrm{SEM}$. $\mathrm{n}=13$ for each group. ROS, rosiglitazone; ROS1, rosiglitazone treatment Group 1 ( $3 \mathrm{mg} \mathrm{kg}^{-1}$ body weight/day; ROS2, rosiglitazone treatment group 2 ( $6 \mathrm{mg} \mathrm{kg}^{-1}$ body weight/day)
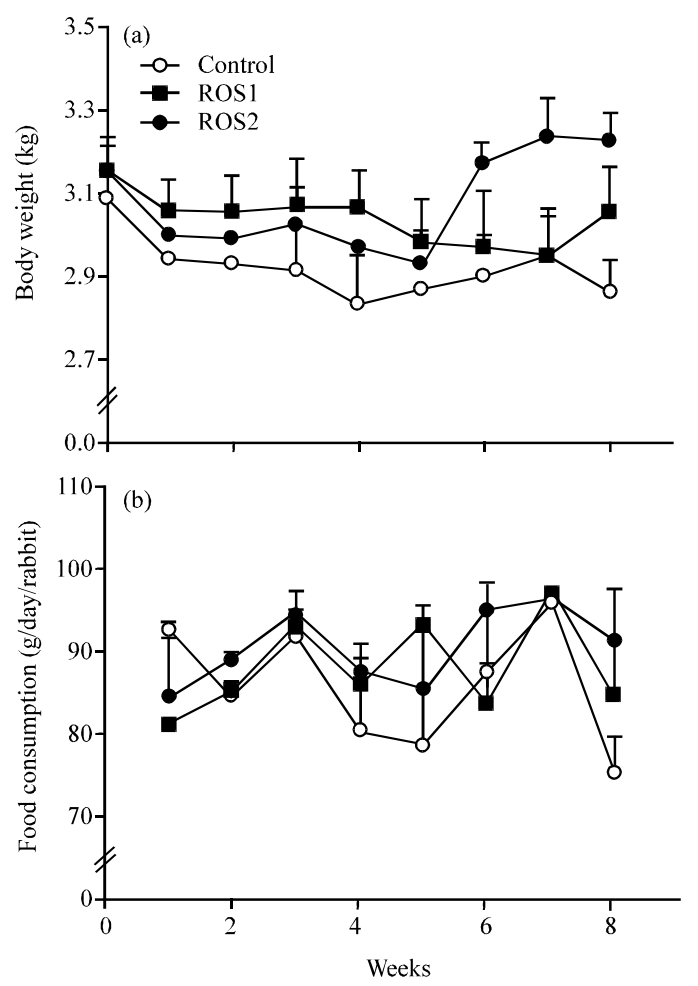

Fig. 2: a) Body weight b) food consumption. Data are expressed as the mean \pm SEM, $n=13$ for each group

three rabbit groups (Fig. 1c and d). An IVGTT was performed in rabbits after feeding HFCD for 8 weeks. Plasma glucose levels in rosiglitazone-treated rabbits were similar to those in control rabbits. There was no significant difference in the AUC of plasma glucose among three rabbit groups.

Evaluation of adipose tissue and organs: As shown in Fig. 2a, the body weight of rosiglitazone-treated rabbits was slightly higher than that of control rabbits but the difference was not statistically significant. The food consumption was also similar among three groups (Fig. 2b). Though the total, visceral and subcutaneous fat weight were higher in the rosiglitazone treatment groups vs the control group, the ratio of fat weight to body weight was also higher in the rosiglitazone treatment groups however, difference was not significant (Fig. 3). Furthermore, there was no significant difference in net weights of the main organs among groups at the end of experiment (Table 1). The ratios of net weight of the main organs to body weight were slightly lower in the rosiglitazone treatment rabbits than that of control rabbits but the difference was not statistically significant (Table 1). 
Pathology and histopathology: The liver color of all rabbits turned yellow and had a greasy consistency. Histological examination revealed that the hepatocyte cytoplasm showed a marked accumulation of fat droplets,
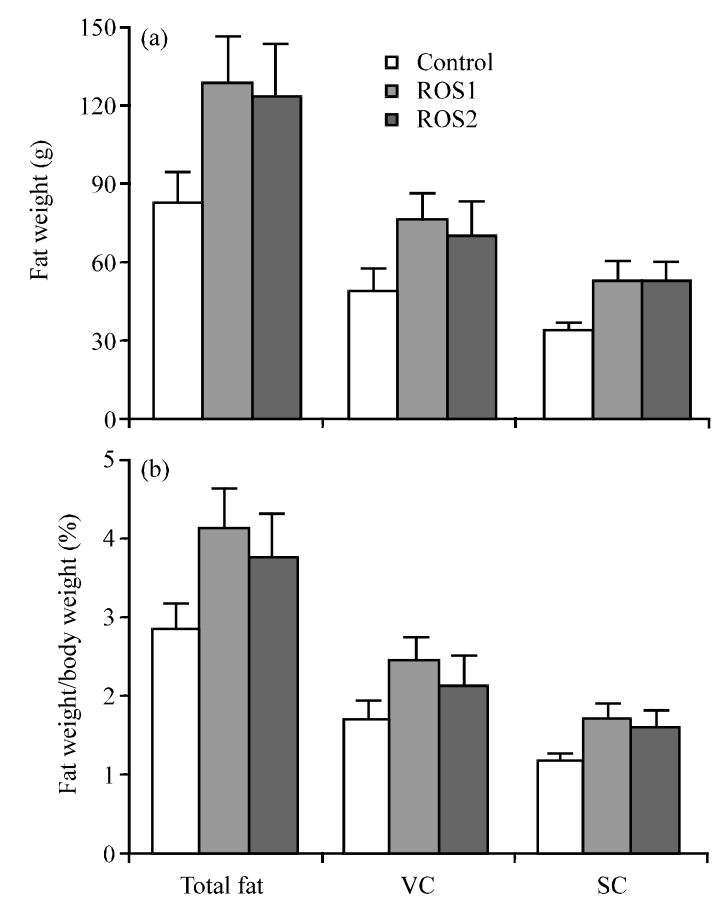

Fig. 3: a) Absolute total, SC and VC fat weight and b) their ratios with body weight. Data are expressed as the mean \pm SEM. SC: Subcutaneous fat; VC: Visceral fat ballooning degeneration and different degrees of liver cell swelling (Fig. 4 d-f). These data and oil red stain results showed that the rabbits NAFLD Model was successfully established in this study. The incidence of serious fatty liver was slightly lower in rosiglitazone treatment rabbits, $20 \%$ (2/10, Group 1), 10\% (1/0, Group 2$)$ versus 33\% (3/9, control group), the degree of liver fatty degeneration of treatment rabbits was not significantly improved by orally administration of rosiglitazone compared with the control rabbits (Table 2).

NAFLD is an acquired metabolic stress-related liver disorder that was originally assumed to be largely confined to residents of affluent industrialized Western

Table 1: Main organ weights and their percentages in body weight

\begin{tabular}{|c|c|c|c|}
\hline Parameters & $\mathrm{CON}$ & ROS 1 & ROS 2 \\
\hline \multicolumn{4}{|c|}{ Organs weight (g) } \\
\hline Liver & $144.24 \pm 7.36$ & $146.36 \pm 7.90$ & $144.94 \pm 8.17$ \\
\hline Heart & $10.66 \pm 1.08$ & $10.13 \pm 0.90$ & $11.54 \pm 0.57$ \\
\hline Kidney & $5.62 \pm 1.21$ & $4.05 \pm 0.63$ & $3.49 \pm 0.64$ \\
\hline Spleen & $18.90 \pm 1.08$ & $17.96 \pm 1.59$ & $19.41 \pm 1.26$ \\
\hline \multicolumn{4}{|c|}{ Per centage in body weight $(\%)$} \\
\hline Liver & $5.04 \pm 0.24$ & $4.78 \pm 0.17$ & $4.46 \pm 0.17$ \\
\hline Heart & $0.67 \pm 0.06$ & $0.59 \pm 0.05$ & $0.60 \pm 0.05$ \\
\hline Kidney & $0.38 \pm 0.05$ & $0.33 \pm 0.03$ & $0.36 \pm 0.02$ \\
\hline Spleen & $0.16 \pm 0.03$ & $0.13 \pm 0.02$ & $0.11 \pm 0.12$ \\
\hline
\end{tabular}

Data are expressed as mean \pm SEM

Table 2: The degree of liver fatty degeneration in three groups

\begin{tabular}{|c|c|c|c|c|c|c|}
\hline \multirow[b]{2}{*}{ Groups } & \multirow[b]{2}{*}{ Numbers } & \multicolumn{5}{|c|}{ The degree of liver fatty degeneration } \\
\hline & & - & + & ++ & +++ & ++++ \\
\hline Control & 9 & 0 & 1 & 3 & 2 & 3 \\
\hline ROS1 & 10 & 0 & 0 & 3 & 5 & 2 \\
\hline ROS2 & 10 & 0 & 1 & 5 & 3 & 1 \\
\hline
\end{tabular}

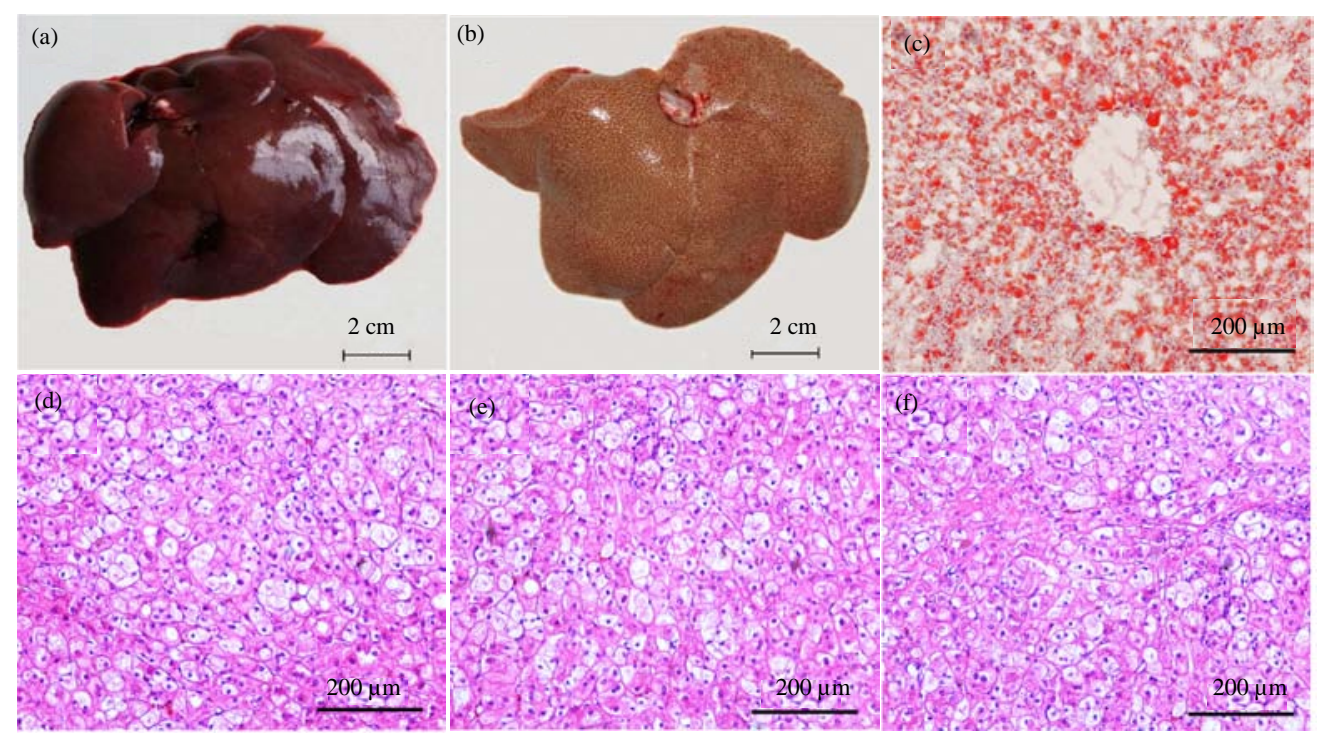

Fig. 4: a) Representative picture of normal liver; b) fatty liver; c) micrographs of accumulation of lipid in oil red stained-liver section, HE stained liver sections from three rabbit groups (d-f) 
countries (Angulo, 2007; Williams, 2006). In the United States, NAFLD affects one in three adults and one in ten children/adolescents (Angulo, 2007). Fatty liver (steatosis) is also highly prevalent in China and is more often linked to obesity than to alcoholism. Among more affluent regions of China, the community prevalence of NAFLD is approximately $15 \%$ (Fan and Farrell, 2009). There is also no standard therapy recommended for NAFLD. Lifestyle modification is currently the initial recommendation but short of recommending diets devoid of processed sugars and saturated fat, the ideal diet is not known (Yki-Jarvinen, 2010; Neuschwander-Tetri, 2009). The main limitation of lifestyle modification is that patients often cannot maintain either dietary changes or exercise habits, so pharmacotherapy is necessary. Obesity and insulin resistance are inextricably linked to NAFLD and therefore therapies directed at weight reduction and improved insulin sensitivity have been investigated.

In this study, a rabbit fatty liver model was successfully induced by HFCD. Rosiglitazone treatment did not significantly change the food consumption, plasma TC, plasma TG, plasma glucose, liver TC and liver TG levels. The histological examination also did not revealed significant differences among the three groups. Though the total body fat weights include visceral fat and subcutaneous fat weights were higher in the rosiglitazone treatment groups vs. the control group, the differences were not statistically significant. Consistent with the findings, weight gain is observed as a side effect of rosiglitazone treatment in human trials. These clinical data showed that rosiglitazone treatment was reasonably well tolerated in patients with inadequately controlled Type 2 diabetes and NAFLD and led to a reduction in aminotransferase levels and improvement in liver histology in patients with nonalcoholic steatohepatitis (Neuschwander-Tetri et al., 2003; Wang et al., 2006). In contrast a study showed that rosiglitazone might not be sufficient in NASH (Ratziu et al., 2010). The same paradoxical results were found in rodent studies (Tahan et al., 2007; Garcia-Ruiz et al., 2007). In this study researchers did not find the beneficial effects in the both doses of rosiglitazone treatment. The effects of rosiglitazone are still disputed regarding cardiovascular effects, especially in recent meta-analysis and epidemiological studies (Home et al., 2007, 2009; Lago et al., 2007). Because of its serious undesired effects, rosiglitazone has been taken off the market in Europe and has restricted use in the US due to a black box warning issued by the Food and Drug Administration (FDA) (Rosen, 2010; Drucker and Goldfine, 2011). In China, rosiglitazone was also restricted by the Chinese state FDA (Chitturi et al., 2002).

\section{CONCLUSION}

The results conclude that rosiglitazone administration might not be sufficient to improve fatty liver in rabbit. The benefits of whether rosiglitazone as a drug for fatty liver disease remain to be considered and additional targets of therapy for liver injury should be explored.

\section{ACKNOWLEDGEMENTS}

This research was partly supported by National Natural Science Grant of China (No.: 30900526 and No.: 8070250), the Fundamental Research Funds for the Central Universities and the China Postdoctoral Science Foundation (20004766).

\section{REFERENCES}

Amarapurka, D.N., A.D. Amarapurkar, N.D. Patel, S. Agal, R. Baigal, P. Gupte and S. Pramanik, 2006. Nonalcoholic steatohepatitis (NASH) with diabetes: Predictors of liver fibrosis. Ann. Hepatol., 5: 30-33.

Angulo, P., 2007. GI epidemiology: Nonalcoholic fatty liver disease. Aliment. Pharmacol. Ther., 25: 883-889.

Bai, L., Y. Jia, N. Viswakarma, J. Huang and A. Vluggens, 2011. Transcription coactivator mediator subunit MED1 is required for the development of fatty liver in the mouse. Hepatology, 53: 1164-1174.

Chan, D.F., A.M. Li, W.C. Chu, M.H. Chan and E.M. Wong et al., 2004. Hepatic steatosis in obese Chinese children. Obes. Rev., 5: 27-42.

Chitturi, S., S. Abeygunasekera, G.C. Farrell, J. HolmesWalker and J.M. Hui et al., 2002. NASH and insulin resistance: Insulin hypersecretion and specific association with the insulin resistance syndrome. Hepatology, 35: 373-379.

Clark, J.M. and A.M. Diehl, 2003. Nonalcoholic fatty liver disease: An underrecognized cause of cryptogenic cirrhosis. JAMA, 289: 3000-3004.

Diehl, A.M., Z. Goodman and K.G. Ishak, 1988. Alcohollike liver disease in nonalcoholics. A clinical and histologic comparison with alcohol-induced liver injury. Gastroenterology, 95: 1056-1062.

Drucker, D.J. and A.B. Goldfine, 2011. Cardiovascular safety and diabetes drug development. Lancet, 377: 977-979.

Fan, J. and T. Watanabe, 2003. Transgenic rabbits as therapeutic protein bioreactors and human disease models. Pharmocol. Ther., 99: 261-282.

Fan, J.G. and G.C. Farrell, 2009. Epidemiology of nonalcoholic fatty liver disease in China. J. Hepatol., 50: $204-210$. 
Folch, J., M. Lees and G.H.S. Stanley, 1957. A simple method for the isolation and purification of total lipides from animal tissues. J. Biol. Chem., 226: 497-509.

Garcia-Ruiz, I., C. Rodriguez-Juan, T. Diaz-Sanjuan, M.A. Martinez, T. Munoz-Yague and J.A. SolisHerruzo, 2007. Effects of rosiglitazone on the liver histology and mitochondrial function in ob/ob mice. Hepatology, 46: 414-423.

Home, P.D., S.J. Pocock, H. Beck-Nielsen, P.S. Curtis and R. Gomis et al., 2009. Rosiglitazone evaluated for cardiovascular outcomes in oral agent combination therapy for type 2 diabetes (RECORD): A multicentre, randomised, open-label trial. Lancet, 373: 2125-2135.

Home, P.D., S.J. Pocock, H. Beck-Nielsen, R. Gomis and M. Hanefeld et al., 2007. Rosiglitazone evaluated for cardiovascular outcomes-an interim analysis. N. Engl. J. Med., 357: 28-38.

Kawai, T., T. Ito, K. Ohwada, Y. Mera, M. Matsushita and $\mathrm{H}$. Tomoike, 2006. Hereditary postprandial hypertriglyceridemic rabbit exhibits insulin resistance and central obesity. A novel model of metabolic syndrome. Arterioscler. Thromb. Vasc. Biol., 26: 2752-2757.

Lago, R.M., P.P. Singh and R.W. Nesto, 2007. Congestive heart failure and cardiovascular death in patients with prediabetes and type 2 diabetes given thiazolidinediones: A meta-analysis of randomised clinical trials. Lancet, 370: 1129-1136.

Liu, E., S. Kitajima, Y. Higaki, M. Morimoto and H. Sun et al., 2005. High lipoprotein lipase activity increases insulin sensitivity in transgenic rabbits. Metabolism, 54: 132-138.

Marchesini, G., E. Bugianesi and G. Forlani, 2003. Nonalcoholic fatty liver, steatohepatitis and the metabolic syndrome. Hepatology, 37: 917-923.

Neuschwander-Tetri, B.A., 2009. Lifestyle modification as the primary treatment of NASH. Clin. Liver Dis., 13: 649-665.

Neuschwander-Tetri, B.A., E.M. Brunt, K.R. Wehmeier, D. Oliver and B.R. Bacon, 2003. Improved nonalcoholic steatohepatitis after 48 weeks of treatment with the PPAR-gamma ligand rosiglitazone. Hepatology, 38: 1008-1017.
Otogawa, K., K. Kinoshita, H. Fujii, M. Sakabe and R. Shiga et al., 2007. Erythrophagocytosis by liver macrophages (Kupffer cells) promotes oxidative stress, inflammation and fibrosis in a rabbit model of steatohepatitis: Implications for the pathogenesis of human nonalcoholic steatohepatitis. Am. J. Pathol., 170: 967-980.

Ratziu, V., F. Charlotte, C. Bernhardt, P. Giral, M. Halbron and G. Lenaour, 2010. Long-term efficacy of rosiglitazone in nonalcoholic steatohepatitis: Results of the fatty liver improvement by rosiglitazone therapy (FLIRT 2) extension trial. Hepatology, 51: 445-453.

Rosen, C.J., 2010. Revisiting the rosiglitazone storylessons learned. N. Engl. J. Med., 363: 803-806.

Tahan, V., F. Eren, E. Avsar, D. Yavuz and M. Yuksel et al., 2007. Rosiglitazone attenuates liver inflammation in a rat model of nonalcoholic steatohepatitis. Dig. Dis. Sci., 52: 3465-3472.

Wang, C.H., C.H. Leung, S.C. Liu and C.H. Chung, 2006. Safety and effectiveness of rosiglitazone in type 2 diabetes patients with nonalcoholic Fatty liver disease. J. Formos. Med. Assoc., 105: 743-752.

Wang, H.N., Y.R. Wang, G.Q. Liu, Z. Liu, P.X. Wu, X.L. Wei and T.P. Hong, 2008. Inhibition of hepatic interleukin-18 production by rosiglitazone in a rat model of nonalcoholic fatty liver disease. World J. Gastroenterol., 14: 7240-7246.

Williams, R., 2006. Global challenges in liver disease. Hepatology, 44: 521-526.

Yki-Jarvinen, H., 2010. Nutritional modulation of nonalcoholic fatty liver disease and insulin resistance: Human data. Curr. Opin. Clin. Nutr. Metab. Care, 13: 709-714.

Zhao, S., Y. Chu, C. Zhang, Y. Lin and K. Xu et al., 2008a. Diet-induced central obesity and insulin resistance in rabbits. J. Anim. Physiol., 92: 105-111.

Zhao, S., C. Zhang, Y. Lin, P. Yang and Q. Yu et al., 2008b. The effects of rosiglitazone on aortic atherosclerosis of cholesterol-fed rabbits. Thromb. Res., 123: 281-287. 